\title{
Antimicrobial susceptibility of large intestinal Escherichia coli isolates from cattle and sheep abattoir samples
}

\author{
Buyukunal EB ${ }^{1 *}$, Mahmood $\mathrm{KH}^{1}$ and Bal $\mathrm{MA}^{2}$ \\ ${ }^{1}$ Department of Biology; ${ }^{2}$ Department of Animal Science, Kahramanmaras Sutcu Imam University, 46100 \\ Kahramanmaras, Turkey
}

\section{Article history}

Received: 23 Aug, 2016

Revised: 6 Sep, 2016

Accepted: 8 Sep, 2016

\begin{abstract}
Escherichia coli reside in gastrointestinal tracts of warm-blooded animals and can serve as an indicator organism for monitoring antibiotic resistance that is considered an important global issue. In this study, E. coli $(\mathrm{n}=49)$ were isolated from 40 samples of colon contents consisting from 20 cattle and 20 sheep carcasses and identified based on standard biochemical tests. The identification was also verified by chromogenic media. All isolates were tested against 13 antibiotic agents by disc diffusion method for determining antimicrobial susceptibilities. Double Disk Synergy test was applied for the detection of the ESBL producing isolates. Nearly all isolates were found to be susceptible to most of the antibiotics. The resistance to tested antibiotics was low; only $6(12.24 \%)$ of $49 \mathrm{E}$. coli isolates were resistant to at least one antibiotic agent. Furthermore, different resistance rates to antibiotics were observed for cattle and sheep isolates. Among 28 isolates from cattle, the highest resistance levels were observed for trimethoprim-sulfamethoxazole $(\mathrm{n}=3 ; 6.12 \%)$, imipenem $(\mathrm{n}=2 ; 4.08 \%)$ and cefpodoxime $(n=1 ; 3.57)$, while only ciprofloxacin resistance $(n=29.52 \%)$ was observed among 21 isolates from sheep. This is the first report about imipenem resistant $E$. coli of animal origin from Turkey. Furthermore, no ESBL positive isolate was detected at all. In conclusion, continuous and strategic surveillance of antimicrobial resistant bacteria in livestock is essential to suppress further dissemination of these bacteria into Turkish society.

Keywords: Abattoir; antibiotics; Enterobacteriaceae; E. coli
\end{abstract}

To cite this article: Buyukunal EB, Mahmood KH and Bal MA, 2016. Antimicrobial susceptibility of large intestinal Escherichia coli isolates from cattle and sheep abattoir samples. Res. Opin. Anim. Vet. Sci., 6(7): 211-217.

\section{Introduction}

Antibiotics have been used extensively for treating bacterial infections in human and veterinary medicine (Smet et al., 2010). They have also been used in poultry production as growth promoter. However, rapid emergence of antibiotic resistant bacteria worldwide is a great concern and limits combating bacterial infections. Therefore, using of antibiotics in veterinary practices, especially in poultry production, has been banned in many countries so that alternative ways have been searched in poultry production.

Cephalosporins from $\beta$-lactam group of antibotics are one of the main groups that are frequently used in both veterinary and human practices. Extended spectrum $\beta$-lactamases (ESBLs) produced by plasmid-

\footnotetext{
*Corresponding author: Buyukunal EB, Kahramanmaras Sutcu Imam University, Department of Biology, Avsar Campus, 46100, Turkey; E-mail: banubal06@gmail.com
} 
encoded genes of bacteria are capable of hydrolyzing penicillins, cephalosporins and monobactams (but not cephamycins and carbapenems) and confer resistance. to $\beta$-lactams. They are usually inhibited by $\beta$-lactamase inhibitors (Bradford, 2001). Expression of ESBLs has been a great concern worldwide and limits the treatment strategies of bacterial infections. It is also well known that plasmids carrying genes for encoding ESBLs may also contain genes encoding resistance to other groups of antibiotics, such as aminoglycosides, fluoroquinolones and folate pathway inhibitors (Paterson et al., 2000).

Escherichia coli occupy the intestinal tract of humans and animals as members of normal flora. Although most strains of $E$. coli are nonpathogenic, some pathogenic strains differ from commensals and express virulence factors that directly involve in pathogenesis (Stender et al., 2001). Besides, cattle have been regarded as a natural reservoir of verotoxin producing E. coli (VTEC) for infections (Mead and Griffin, 1998). The organism is carried in the gastrointestinal tract and is discarded to the feces (Bach et al., 2004). E. coli $\mathrm{O} 157$ was detected more frequently in the colon than in the rumen of slaughtered healthy cattle (Laven et al., 2003). E. coli O157:H7 can be transmitted to carcass surfaces when gut contents or fecal materials get in contact with meat surfaces during the processes of skinning and evisceration (Dickson and Anderson, 1992). The same transmission route can also be effective for dissemination of the antibiotic resistant commensal E. coli isolates.

The studies on the prevalence of antibiotic resistant of $E$. coli in rectum contents and/or feces of slaughtered animals have been documented more frequently around the world as well in Turkey (Kucukbasmaci et al., 2008; Mustak et al., 2012; Amosun et al., 2012; Wasyl et al. 2013). There is no report on antibiotic susceptibility patterns of $E$. coli recovered from colon contents of healthy food producing animals slaughtered in Turkey. For this reason, we aimed to monitor prevalence of antibiotic resistant E. coli isolates from colon contents of sheep and cattle slaughtered in Kahramanmaras province of Turkey. In addition, prevalence of ESBL producing isolates was also addressed.

\section{Materials and Methods}

\section{Sample collection}

The experimental procedure of this study was approved by The Faculty of Agriculture, Animal Policy and Welfare Committee of the Kahramanmaras Sutcu Imam University (protocol number: 2013/08-1). Colon contents were sampled from slaughtered cattle and sheep daily. Viscera of slaughtered animals were separated in a special place right away after evisceration. Forty colon samples (20 from cattle and 20 from sheep) were collected immediately and aseptically before cleaning process of the viscera by workers. The aseptic sampling process was done by making a small incision in colon wall with a sterile blade and insertion of a sterile cotton swab through the colon wall to get intestinal contents, then replacing the swab into the sterile disposable culture tube containing $1 \mathrm{ml}$ of sterile 1X Phosphate Buffered Saline (PBS) at $\mathrm{pH}$ 7.4. Buffer was contained $137 \mathrm{mmol}$ of $\mathrm{NaCl}$, $2.7 \mathrm{mmol} \mathrm{g}$ of $\mathrm{KCl}, 10 \mathrm{mmol}$ of $\mathrm{Na}_{2} \mathrm{HPO}_{4} \cdot 2 \mathrm{H}_{2} \mathrm{O}$ and $2 \mathrm{mmol}$ of $\mathrm{KH}_{2} \mathrm{PO}_{4}$ per $\mathrm{L}$. The collected samples were immediately transported and processed for bacteriological examination upon arrival in the laboratory.

\section{Bacteriological analyses}

Bacterial inoculation was initially made on EosinMethylene Blue (EMB) agar. Afterward, inoculated plates were incubated at $35^{\circ} \mathrm{C}$ for 24 hours. At the end of incubation, the plates were examined for selection of typical E. coli colonies (dark-centered and flat, with or without metallic sheen) and re-streaked on fresh EMB agar for purity. Grown pure isolates were transferred into Tryptic Soy Broth (TSB) and were grown at $35^{\circ} \mathrm{C}$ for 24 hours. Grown cultures were then used for preparation of long-term bacterial stocks containing $15 \%$ of sterile glycerol at the final volume.

\section{Identification of $E$. coli strains}

Identification of $E$. coli strains was done based on conventional biochemical tests including oxidase, triple sugar iron (TSI), lysine decarboxylase, indole, methyl red, Voges-Proskauer and citrate. Biochemical identification of the bacterial isolates was further verified using CHROMagar ECC (France). All E. coli colonies appeared in blue color on this selective medium.

\section{Antimicrobial susceptibility test}

Antimicrobial susceptibilities of the isolates were determined by Kirby-Bauer Disk Diffusion Susceptibility Test protocol as described by CLSI (2013). Thirteen different antimicrobial containing disks (Oxoid, U.K) were used for the testing susceptibilities of $E$. coli isolates. Antimicrobial agent concentrations on each disk were as follows: amoxicillin-clavulanic acid $(30 \mu \mathrm{g})$, piperacillintazobactam $(110 \mu \mathrm{g})$, ceftazidime $(30 \mu \mathrm{g})$, cefotaxime $(30 \mu \mathrm{g})$, cefpodoxime $(10 \mu \mathrm{g})$, ceftriaxone $(30 \mu \mathrm{g})$, ertapenem $(10 \mu \mathrm{g})$, imipenem $(10 \mu \mathrm{g})$, meropenem $(10$ $\mu \mathrm{g})$, aztreonam $(30 \mu \mathrm{g})$, ciprofloxacin $(5 \mu \mathrm{g})$, amikacin $(30 \mu \mathrm{g})$ and trimethoprim-sulphamethoxazole $(25 \mu \mathrm{g})$. E. coli ATCC 25922 was used as quality control strain. Results were interpreted according to Clinical and Laboratory Standards Institute criteria (CLSI, 2013). 


\section{Double disk synergy test}

Detection of ESBL producing isolates was performed with double disk synergy test using the following antibiotic disks: amoxicillin/clavulanic acid (30 $\mu \mathrm{g})$, aztreonam $(30 \mu \mathrm{g})$, cefotaxime $(30 \mu \mathrm{g})$, ceftazidime $\left(\begin{array}{ll}30 & \mu \mathrm{g}\end{array}\right)$, ceftriaxone $\left(\begin{array}{lll}30 & \mu \mathrm{g}\end{array}\right)$ and cefpodoxime (10 $\mu \mathrm{g})$ (Oxoid, UK) (CLSI, 2013). Briefly, inoculums were prepared in sterile saline solutions from grown cultures. When $0.5 \mathrm{McFarland}$ turbidity value was obtained for each bacterial inoculum, they were inoculated onto Mueller Hinton Agar (Merck) by sterile cotton swab. Then, amoxicillin/clavulanic acid was placed at the center and the others around it that were far from each other (24 $\mathrm{mm}$ from center to center). Subsequently, they were incubated at $37^{\circ} \mathrm{C}$ for $18-20$ hours. A clear extension or protrusion (synergistic effect) of the edge of the inhibition zone of any of the antibiotics toward the disk containing amoxicillin/clavulanic acid was interpreted as positive for ESBL production. Three clinical isolates of ESBL-producing E. coli, possessing bla $a_{\mathrm{TEM}}, b_{\mathrm{SHV}}$ and bla $a_{\mathrm{CTX}}-\mathrm{M} \beta$-lactamase genes, were used as control strains during double disk synergy test (Durmaz et al., 2015).

\section{Results}

Forty-nine E. coli isolates were recovered from 34 out of 40 (20 from cattle and 20 from sheep) colon contents. Of them, 28 (57.14\%) were recovered from 19 cattle samples and $21(42.85 \%)$ were isolated from 15 sheep samples. As a result, E. coli was detected from 19 (95\%) of cattle and $15(75 \%)$ of sheep samples. When considering antibiotic susceptibilities of all E. coli isolates, resistance was observed only for four antibiotics (trimethoprim-sulfamethoxazole, imipenem, cefpodoxime and ciprofloxacin) (Figure 1). Moreover, none of the $E$. coli isolates from cattle samples was resistant to ciprofloxacin. Similarly, no isolates from sheep samples were resistant to trimethoprim- sulfamethoxazole, imipenem, cefpodoxime (Tables 1 and 2). In addition, the highest resistance level of cattle isolates was found for trimethoprim-sulfamethoxazole $(10.71 \%)$ and highest resistance level of sheep isolates was seen for ciprofloxacin $(9.52 \%)$. Of all $E$. coli isolates from cattle and sheep samples, 6 (12.24\%) were resistant to at least one antibiotic agent. Moreover, number of resistant isolates was higher among cattle isolates (6) than sheep isolates (2). Of $28 \mathrm{E}$. coli isolates from cattle samples, $4(14.28 \%)$ were resistant to at least one antibiotic agent. Of $21 \mathrm{E}$. coli isolates from sheep samples, $2(9.52 \%)$ were resistant to at least one antibiotic agent. So, resistant isolates were detected in higher frequency among cattle isolates.

Out of $49 \mathrm{E}$. coli isolates from cattle and sheep samples, $2(4.08 \%)$ were found as multiple drug resistant displaying resistance to two antibiotics. Multiple resistant isolates were isolated only from cattle samples, and they were resistant to both imipenem and trimethoprim-sulfamethoxazole (Table 1). Imipenem (carbapenem) resistant E. coli from cattle origin was detected as the first time in Turkey with this study. Moreover, one cattle isolate was resistant to cefpodoxime. This isolate had also selected as presumably ESBL producer in the initial screening. However, it was classified as ESBL negative based on the results of double disk synergy test. Therefore, none of the isolates were ESBL positive.

It is noteworthy that intermediate level of resistance was quite high for most of the tested antibiotics for both cattle and sheep isolates. Intermediate resistance was observed for ten out of thirteen antibiotics (piperacillin-tazobactam, ceftazidime, cefotaxime, cefpodoxime, ertapenem, imipenem, aztreonam, ciprofloxacin, meropenem, amikacin, ertapenem) among cattle isolates, while it was observed for six antibiotics (piperacillintazobactam, ceftazidime, cefotaxime, cefpodoxime, imipenem, amikacin, ertapenem, ertapenem) among sheep isolates (Tables 1 and 2).

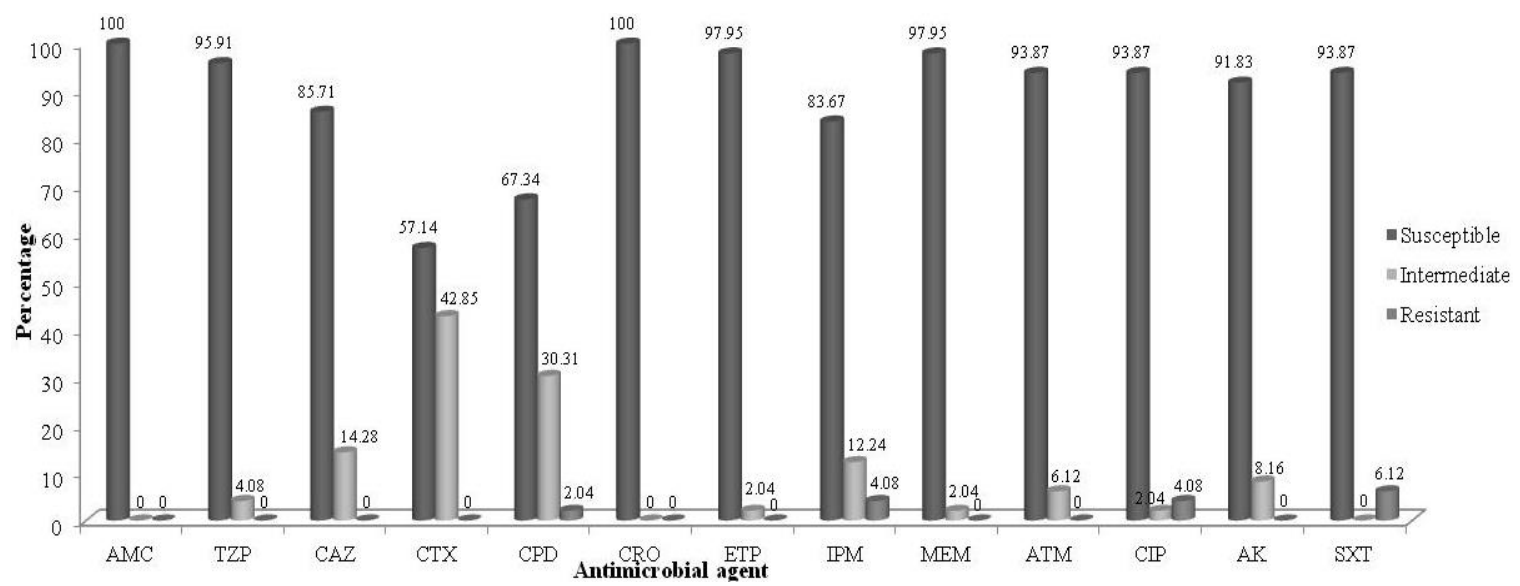

Fig. 1: Antimicrobial susceptibility patterns of all $E$. coli isolates from cattle and sheep colon contents. 
Table 1: Antimicrobial susceptibilities of $E$. coli isolates from cattle colon contents

\begin{tabular}{|c|c|c|c|}
\hline \multirow[t]{2}{*}{$\begin{array}{l}\text { Antimicrobial } \\
\text { agent }\end{array}$} & \multirow{2}{*}{$\begin{array}{c}\text { Susceptible } \\
\mathrm{n}(\%)\end{array}$} & \multirow{2}{*}{$\begin{array}{c}\text { Intermediate } \\
\mathrm{n}(\%)\end{array}$} & \multirow{2}{*}{$\frac{\text { Resistant }}{\mathrm{n}(\%)}$} \\
\hline & & & \\
\hline AMC & $28(100)$ & $0(0)$ & $0(0)$ \\
\hline $\mathrm{TZP}$ & $27(96.42)$ & $1(3.57)$ & $0(0)$ \\
\hline CAZ & $24(85.71)$ & $4(14.28)$ & $0(0)$ \\
\hline CTX & $13(46.42)$ & $15(53.57)$ & $0(0)$ \\
\hline $\mathrm{CPD}$ & $16(57.14)$ & $11(39.28)$ & $1(3.57)$ \\
\hline $\mathrm{CRO}$ & $28(100)$ & $0(0)$ & $0(0)$ \\
\hline ETP & $27(96.42)$ & $1(3.57)$ & $0(0)$ \\
\hline IPM & $22(78.57)$ & $4(14.28)$ & $2(7.14)$ \\
\hline MEM & $27(96.42)$ & $1(3.57)$ & $0(0)$ \\
\hline ATM & $25(89.28)$ & $3(10.71)$ & $0(0)$ \\
\hline CIP & $27(96.42)$ & $1(3.57)$ & $0(0)$ \\
\hline $\mathrm{AK}$ & $26(92.85)$ & $2(7.14)$ & $0(0)$ \\
\hline SXT & $25(89.28)$ & $0(0)$ & $3(10.71)$ \\
\hline \multicolumn{4}{|c|}{$\begin{array}{l}\text { AMC, amoxicillin-clavulanic acid; TZP, piperacillin } \\
\text { tazobactam; CAZ, ceftazidime; CTX, cefotaxime; CPD, } \\
\text { cefpodoxime; CRO, ceftriaxone; ETP, ertapenem; IPM, } \\
\text { imipenem; MEM, meropenem; ATM, aztreonam; CIP, } \\
\text { ciprofloxacin; AK, amikacin; SXT, trimethoprim- } \\
\text { sulphamethoxazole. }\end{array}$} \\
\hline
\end{tabular}

Table 2: Antimicrobial susceptibilities of $E$. coli isolates from sheep colon contents

\begin{tabular}{lccc}
\hline \multirow{2}{*}{$\begin{array}{l}\text { Antimicrobial } \\
\text { agent }\end{array}$} & Susceptible & Intermediate & Resistant \\
\cline { 2 - 4 } AMC $(\%)$ & $\mathrm{n}(\%)$ & $\mathrm{n}(\%)$ \\
TZP & $21(100)$ & $0(0)$ & $0(0)$ \\
CAZ & $20(95.23)$ & $1(4.76)$ & $0(0)$ \\
CTX & $18(85.71)$ & $3(14.28)$ & $0(0)$ \\
CPD & $15(71.42)$ & $6(28.57)$ & $0(0)$ \\
CRO & $17(80.95)$ & $4(19.04)$ & $0(0)$ \\
ETP & $21(100)$ & $0(0)$ & $0(0)$ \\
IPM & $21(100)$ & $0(0)$ & $0(0)$ \\
MEM & $19(90.47)$ & $2(9.52)$ & $0(0)$ \\
ATM & $21(100)$ & $0(0)$ & $0(0)$ \\
CIP & $21(100)$ & $0(0)$ & $0(0)$ \\
AK & $19(90.47)$ & $0(0)$ & $2(9.52)$ \\
SXT & $19(90.47)$ & $2(9.52)$ & $0(0)$ \\
AMC & $21(100)$ & $0(0)$ & $0(0)$ \\
\hline
\end{tabular}

AMC, amoxicillin-clavulanic acid; TZP, piperacillintazobactam; CAZ, ceftazidime; CTX, cefotaxime; CPD, cefpodoxime; CRO, ceftriaxone; ETP, ertapenem; IPM, imipenem; MEM, meropenem; ATM, aztreonam; CIP, ciprofloxacin; AK, amikacin; SXT, trimethoprimsulphamethoxazole.

\section{Discussion}

There are several reports about the prevalence of $E$. coli in healthy food producing ruminants, such as cattle and sheep at slaughter worldwide. For example, a study conducted in Portugal, 192 E. coli isolates from 198 fecal samples were detected from rectal samples of healthy food animals at slaughter. These include pig ( $\mathrm{n}$ $=66 ; 34.37 \%)$, sheep $(\mathrm{n}=73 ; 38.02 \%)$ and cattle $(\mathrm{n}=$ 53; 27.60\%) isolates (Ramos et al. 2013). This reveals that occurrence of $E$. coli in cattle $(\mathrm{n}=19 ; 95 \%)$ and sheep samples $(\mathrm{n}=15 ; 75 \%)$ was higher in our findings than the findings by Ramos et al. (2013).

Up to now, there is no report about using CHROMagar ECC for identifying bacteria of animal origin in Turkey to our knowledge, whereas there are several reports on using CHROMagar ECC for samples of animal origin worldwide. For instance, Liebana et al. (2006) and Hasan and Tinni (2011) observed blue colonies of E. coli isolates on CHROMagar ECC from animal sources. Gosling et al. (2012) reported blue colonies of $E$. coli isolated from turkeys in Great Britain, as well.

The resistant E. coli isolates from cattle and sheep samples were much less than the susceptible ones in the present study and were consistent to results from previous studies around the world. For example, in one of the most recent study conducted in Poland indicated that $79.9 \%$ of cattle E. coli isolates that most were originated from adult cattle showed no resistance (Wasyl et al., 2013). This might be presumed that some animals were slaughtered due to insufficient milk productivity and remain un-treated with antimicrobials due to restriction on milk production during withdrawal period. On the opposite, the higher resistance levels detected in broiler $(5.1 \%)$ and turkey $(11.3 \%)$ isolates might be explained with longer antibiotic treatment of poultry until few days before slaughter. The higher resistance in $E$. coli isolates from pig was also noticed when compared to cattle and sheep isolates worldwide. For example, the study conducted in Great Britain stated that out of $836 \mathrm{E}$. coli isolates tested only 5.7\% from cattle were resistant to one or more antimicrobials, while only $3.0 \%$ of 836 isolates from sheep were resistant to one or more agents. However, $92.1 \%$ of 2480 isolates from pigs were resistant to at least one antimicrobial (Enne et al., 2008). Such variation may reflect the differences in the amounts of therapeutic antimicrobials used in the husbandry of the three animal species studied. Similar findings were documented from Korea by Lim et al. (2007) which prevalence of resistance in $E$. coli isolates of pigs was much higher than that in cattle, with $98.3 \%$ of pig isolates and $37.1 \%$ of cattle isolates showing resistance to one or more of the antimicrobial agents tested.

Several studies addressed antibiotic resistances among Enterobacteriaceae isolates obtained from foods of animal origin such as minced-meat, chicken, cheese and ice cream in Turkey. In one of them, it was found that resistance among $72 \mathrm{E}$. coli isolates occurred more frequently for erythromycin $(91.7 \%)$, tetracycline (55.6\%), ampicillin (50\%), sulphamethoxazole/ trimethoprim (45.8\%) and piperacillin (41.7\%) (Arslan and Eyi, 2010). In another study, 45 E. coli isolates were recovered from different food samples including raw calf meat (minced), chicken meat, raw milk, white cheese and ice cream collected from various 
supermarkets, dairy plants and pastry shops (Gundogan and Avci, 2013). Antibiotic susceptibilities of those isolates indicated that they were resistant to cefotaxime (33.3\%), ciprofloxacin $(31.1 \%)$, aztroenam $(28.9 \%)$, ceftazidime $(8.9 \%)$, ceftriaxone $(8.9 \%)$, amoxicillin/ clavulanic acid (6.7\%), and amikacin (4.4\%). Moreover, all isolates have been found sensitive to imipenem, ertapenem and piperacillin/tazobactam. In contrast to those studies, out of $49 \mathrm{E}$. coli isolates in the present study, resistance to cefpodoxime, imipenem, ciprofloxacin and trimethoprim-sulfamethoxazole was detected at much lower levels; 1 (2.04\%), 2 (4.08\%), 2 $(4.08 \%)$ and $3(6.12 \%)$, respectively. Finding lower level of resistances in the present study might be conceivable when considering all food originated samples have been gone through various processes such as slaughtering, handling and packaging which might introduce more resistant bacteria.

Ciprofloxacin is a broad-spectrum fluoroquinolone antimicrobial agent that is one of the most effective antibiotics for treatment of various infections in humans and animals. Compared to our study $(4.08 \%)$, the observed resistances of food originated E. coli isolates to ciprofloxacin were much higher; $31.9 \%$ and $31.1 \%$, respectively from other studies (Arslan and Eyi 2011; Gundogan and Avci, 2013). However, the few most recent studies from Turkey focused on fluoroquinolones (ciprofloxacin) resistant E. coli isolated from animals (Cengiz et al., 2012; Mustak et al., 2012) and reported that $47(50.0 \%)$ of 94 chicken, 3 (4.5\%) of 66 sheep, 5 (9.6\%) of 52 cattle and $3(6.4 \%)$ of 47 dog E. coli strains were found to be resistant to ciprofloxacin. In contrast to that study, a low level of ciprofloxacin resistance $(9.52 \%)$ detected in the present study for sheep isolates.

The current worldwide emergence of resistance to the powerful antibiotic carbapenem in Enterobacteriaceae constitutes an important growing public health threat (Nordmann et al., 2012). A recent report from Turkey indicated a need for both continued surveillance and prudent use of the $\beta$-lactam (carbapenems) due to occurrence of carbapenem resistant isolates (two E. coli, one K. pneumoniae and one $K$. oxytoca) from clinical specimens of hospitalized patients and outpatients (Kuzucu et al., 2011). However, carbapenem-resistant bacteria or carbapenemase-producing isolates from animals especially in cattle were reported in few studies from different countries in the last five years (Webb et al. 2016). Imipenem resistant E. coli isolates (n=2) was detected in cattle samples for the first time with our study from Turkey. In the present study, two imipenem resistant isolates were also resistant to trimethoprimsulphamethoxazole pointing out multiple resistant feature of those isolates which is in accordance with the results of a previous study (Kucukbasmaci et al., 2008).
During the last decade, extended-spectrum $\beta$ lactamase (ESBL) producing Enterobacteriaceae have become a matter of great concern in human medicine. The presence of ESBL-producing strains in the community raises a question about possible reservoirs. Recent studies describe the occurrence of ESBLproducing Enterobacteriaceae in meat, fish, and raw milk. Therefore, the impact of food animals as reservoirs and disseminators of such strains into the food production chain must be assessed (Geser et al. 2011). According to previous studies, ESBLs producing Enterobacteriaceae have been detected more frequently among $E$. coli isolates recovered from foods of animal origin in Turkey. Arslan and Eyi (2011) reported that $13.9 \%$ of E. coli isolates produced ESBL. Gundogan and Avci (2013) observed a higher prevalence (44.4\%) for ESBL production among $45 \mathrm{E}$. coli isolates. So far, a single report existed about ESBL producing Enterobacteriaceae isolated from healthy food producing animals in Turkey and indicated detection of 5 ESBL-producing Enterobacteriaceae isolates from cattle; comprised from $3 \mathrm{E}$. coli and 2 Citrobacter species, out of 349 samples $(2.1 \%)$ from fecal samples of various animals (cattle, calves, sheep) collected from three different slaughterhouses and two farms (Kucukbasmaci et al., 2008). Kucukbasmaci et al. (2008) also used PCR and sequencing for determining of genetic determinants that are responsible for the ESBL phenotypes and found that all of the five isolates harbored TEM-1 and SHV (four isolates SHV-5 and one isolate SHV-12) type $\beta$-lactamases. Following to that study, our study is considered as the second attempt for detection of ESBL positive Enterobacteriaceae in healthy food animals at slaughter from Turkey.

Reports addressing the prevalence of ESBLproducing $E$. coli in food-producing animals revealed variable rates that are ranged from $0.2 \%$ to $40.1 \%$ in different countries according to the country and the methodology (Smet et al., 2010). Duan et al. (2006) noticed prevalence of ESBL producers as 3.1\% among E. coli isolates from fecal samples of healthy cattle in Hong Kong, whereas higher prevalence $(17.1 \%)$ of ESBL-producing Enterobacteriaceae, mostly E. coli, was detected from bovine fecal samples in Switzerland (Geser et al., 2011). However, as common, most recent reports show increasing detection rate of ESBLs producing bacteria in most countries. At this point, comparison of prevalence data from other countries is difficult due to application of different methodologies. (Smet et al., 2010). Moreover, prevalence might be related to preferences of most frequently used antimicrobial agents in veterinary practices for particular country. For instance, ceftiofur can easily be purchased even without a veterinary prescription in Turkey and unfortunately, reliable data is lacking on the antimicrobial agent consumption in veterinary medicine in Turkey (Kucukbasmaci et al., 2008). 
Many studies proved that antibiotic resistant bacteria found in livestock can be a reservoir for transmission of resistant bacteria into humans (Shiraki et al., 2004; Kluytmans et al., 2013). For this reason, caution should be delivered to antibiotic resistant bacteria in animals for public health concerns. In conclusion, even though relatively low level of resistance was detected for $E$. coli isolates of cattle and sheep in the present study, levels are still significant. Therefore, especially multidrug resistant and ESBLproducing $E$. coli species should be monitored regularly in animals.

\section{Acknowledgements}

This research was supported by Kahramanmaras Sutcu Imam University Grant (2013/6-17 YLS).

\section{References}

Amosun, EA, Ojo OE, Alao IK, Ajuwape ATP (2012) Antimicrobial resistance among commensal Escherichia coli from cattle faeces and beef in Ibadan, Nigeria. Afr J Biotechnol 11:12240-12245.

Arslan S, Eyi A (2011) Antimicrobial resistance and ESBL prevalence in Escherichia coli from retail meats. J Food Safety 31: 262-267.

Dickson JS, Anderson ME (1992) Microbiological decontamination of food animal carcasses by washing and sanitizing systems: A review. J Food Protect 55:133-140.

Durmaz S, Buyukunal Bal EB, Gunaydin M, Yula E, Percin D (2015) Detection of $\beta$-lactamase genes, ERIC-PCR typing and phylogenetic groups of ESBL producing quinolone resistant clinical Escherichia coli isolates. Biomed Res 26: 43-50.

Bach SJ, McCallister TA, Mears GJ, SchwartzkopfGenswein KS (2004) Long-haul transport and lack of preconditioning increases fecal shedding of Escherichia coli and Escherichia coli O157:H7. J Food Protect 67: 672-678.

Bradford PA (2001) Extended-spectrum $\beta$-lactamases in the 21st century: Characterization, epidemiology, and detection of this important resistance threat. Clin Microbiol Rev 14: 933-951.

Cengiz M, Buyukcangaz E, Arslan E, Mat B, Sahinturk P, Sonal S, Gocmen H, Sen A (2012) Molecular characterisation of quinolone resistance in Escherichia coli from animals in Turkey. Vet Rec 11: 171(6): 155, 1-4.

CLSI, 2013. Clinical and Laboratory Standards Institute: Performance standards for Antimicrobial Susceptibility Testing; Twenty-Third Informational Supplement. CLSI Document M100-S23, Wayne, PA 19087, USA. 33(1): 44-50.

Duan RS, Sit TH, Wong SS, Wong RC, Chow KH Mak GC, Yam WC, Ng LT, Yuen KY, Ho PL (2006)
Escherichia coli producing CTX-M $\beta$-lactamases in food animals in Hong Kong. Microb Drug Resist 12: $145-148$.

Enne VI, Cassar C, Sprigings K, Woodward MJ, Bennett PM (2008) A high prevalence of antimicrobial resistant Escherichia coli isolated from pigs and a low prevalence of antimicrobial resistant $E$. coli from cattle and sheep in Great Britain at slaughter. FEMS Microbiol Lett 278: 193-199.

Geser N, Stephan R, Kuhnert P, Zbinden R, Kaeppeli U, Cernela N, Haechler H (2011) Fecal carriage of extended-spectrum $\quad \beta$-lactamase-producing Enterobacteriaceae in swine and cattle at slaughter in Switzerland. J Food Protect 74: 446-449.

Gosling RJ, Clouting CS, Randall LP, Horton RA, Davies RH (2012) Ciprofloxacin resistance in $E$. coli isolated from Turkeys in Great Britain. Avian Pathol 41: 83-89.

Gundogan N, Avci E (2013) Prevalence and antibiotic resistance of extended-spectrum beta-lactamase (ESBL) Producing Escherichia coli and Klebsiella species isolated from foods of animal origin in Turkey. Afr J Microbiol Res 7: 4059-4064.

Hasan MM, Tinni SR (2011) Detection of integrons from Escherichia coli isolates obtained from some selected animals in Republic of Korea. Indian $\mathbf{J}$ Microbiol 51: 144-146.

Kluytmans JA, Overdevest IT, Willemsen I, Kluytmansvan den Bergh MF, Van der Zwaluw K, Heck M, Rijnsburger M, Vandenbroucke-Grauls CM, Savelkoul PH, Johnston BD, Gordon D, Johnson JR (2013) Extended-spectrum $\beta$-lactamase producing Escherichia coli from retail chicken meat and humans: Comparison of strains, plasmids, resistance genes, and virulence factors. Clin Infect Dis 56: 478-487.

Kucukbasmaci, O, Ciftcioglu G, Midilli K, Issa G (2008) Detection of extended spectrum $\beta$-lactamase producing Enterobacteriaceae from food animals in Turkey. Rev Med Vet 159: 586-592.

Kuzucu C, Yetkin F, Görgec S, Ersoy Y (2011) Investigation of the susceptibilities of extendedspectrum beta-lactamase-producing Escherichia coli and Klebsiella spp. strains to ertapenem and other carbapenems. Mikrobiyol Bul 45: 28-35.

Laven RA, Ashmore A, Stewart CS (2003) Escherichia coli in the rumen and colon of slaughter cattle, with particular reference to E. coli O157. Vet J 165: 7883.

Liebana E, Batchelor M, Hopkins KL, Clifton-Hadley FA, Teale CJ, Foster A, Barker L, Threlfall EJ, Davies RH (2006) Longitudinal farm study of extended-spectrum $\beta$-lactamase-mediated resistance. J Clin Microbiol 44: 1630-1634. 
Lim SK, Lee HS, Nam HM, Cho YS, Kim JM, Song SW, Park YH, Jung SC (2007) Antimicrobial resistance observed in Escherichia coli strains isolated from fecal samples of cattle and pigs in Korea during 2003-2004. Int J Food Microbiol 116: 283-286.

Mead PS, Griffin P (1998) Escherichia coli O157:H7. Lancet 352: 1207-1212.

Mustak HK, Ica T, Ciftci A, Diker KS (2012) Plasmidmediated quinolone resistance in Escherichia coli strains isolated from animals in Turkey. Ankara Univ Vet Fak 59: 255-258.

Nordmann P, Dortet L, Poirel L (2012) Carbapenem resistance in Enterobacteriaceae: Here is the storm. Trends Mol Med 18: 263-272.

Paterson DL, Mulazimoglu L, Casellas JM, Ko WC, Goossens H, Von Gottberg A, Mohapatra S, Trenholme GM, Klugman KP, McCormack JG, Yu VL (2000) Epidemiology of ciprofloxacin resistance and its relationship to extended-spectrum $\beta$ lactamase production in Klebsiella pneumoniae isolates causing bacteremia. Clin Infect Dis 30: 473-478.

Ramos S, Silva N, Caniça M, Capelo Martinez JL, Brito F, Igrejas G, Poeta P (2013) High prevalence of antimicrobial-resistant Escherichia coli from animals at slaughter: A food safety risk. J Sci Food Agr 93: 517-526.

Shiraki Y, Shibata N, Doi Y, Arakawa Y (2004) Escherichia coli producing CTX-M-2 beta-lactamase in Cattle, Japan. Emerg Infect Dis 10: 69-75.

Smet A, Martel A, Persoons D, Dewulf J, Heyndrickx M, Herman L, Haesebrouck F, Butaye P (2010) Broad-spectrum $\quad \beta$-lactamases among Enterobacteriaceae of animal origin: Molecular aspects, mobility and impact on public health. FEMS Microbiol Rev 34:295-316.

Stender H, Oliveira K, Rigby S, Bargoot F, Coull J (2001) Rapid detection, identification, enumeration of Escherichia coli by fluorescence in situ hybridization using an array scanner. J Microbiol Methods 45: 31-39.

Wasyl D, Hoszowski A, Zając M, Szulowski K (2013) Antimicrobial resistance in commensal Escherichia coli isolated from animals at slaughter. Front Microbiol 4: 221

Webb HE, Bugarel M, den Bakker HC, Nightingale KK, Granier SA, Scott HM, Loneragan GH (2016) Carbapenem-resistant bacteria recovered from faeces of dairy cattle in the high plains region of the USA. PLoS ONE 11: e0147363. doi:10.1371/journal.pone.0147363. 